\title{
Pharmacogenomic response of low dose haloperidol in critically ill adults with delirium
}

\author{
Zoran Trogrlić, RN, PhD ${ }^{\mathrm{a}, *}$, Mathieu van der Jagt, $\mathrm{MD}, \mathrm{PhD}^{\mathrm{a}}$, Robert Jan Osse, $\mathrm{MD}, \mathrm{PhD}^{\mathrm{b}}$, \\ John W. Devlin, PharmD, FCCM ${ }^{\mathrm{c}}$, Daan Nieboer, MSc ${ }^{\mathrm{d}}$, Birgit C.P. Koch, PhD ${ }^{\mathrm{e}}$, \\ Ron H.N. van Schaik, PhD ${ }^{\text {f }}$, Nicole G.M. Hunfeld, PharmD ${ }^{\text {a,e }}$ \\ a Department of Intensive Care Adults, Erasmus MC University Medical Center, Doctor Molewaterplein 40, 3015 GD Rotterdam, the Netherlands \\ b Department of Psychiatry, Erasmus MC University Medical Center, Doctor Molewaterplein 40, 3015 GD Rotterdam, the Netherlands \\ c School of Pharmacy, Northeastern University, 360 Huntington Ave, Boston, MA 02115, USA \\ d Department of Public Health, Erasmus MC University Medical Center Rotterdam, Doctor Molewaterplein 40, 3015 GD Rotterdam, the Netherlands \\ e Department of Hospital Pharmacy, Erasmus MC University Medical Center, Doctor Molewaterplein 40, 3015 GD Rotterdam, the Netherlands \\ ${ }^{\mathrm{f}}$ Department of Clinical Chemistry, Erasmus University Medical Center, Doctor Molewaterplein 40, 3015 GD Rotterdam, the Netherlands
}

\section{A R T I C L E I N F O}

Available online $\mathrm{xxxx}$

Keywords:

Delirium

Intensive care

Haloperidol

Pharmacogenetics

Pharmacokinetics

Pharmacodynamics

\begin{abstract}
A B S T R A C T
Purpose: To characterize the pharmacogenomic response of low-dose haloperidol for delirium treatment in critically ill adults.

Materials and methods: Single-center, pilot study of a convenience sample of ICU adults with delirium treated with low-dose IV haloperidol. Patients were evaluated for delirium with the ICDSC every $8 \mathrm{~h}$. Serum haloperidol concentrations were collected on ICU days 2-6, CYP2D6 and CYP3A4 genotypes were characterized and patients were categorized as extensive (EM), intermediate (IM) or poor metabolizers (PM).

Results: The 22 patients (median age 67 [IQR 48,77] years; median APACHE III 81[IQR 54,181]; CYP2D6 [EM = 12, $\mathrm{IM}=7, \mathrm{PM}=3], \mathrm{CYP3A}[\mathrm{EM}=18, \mathrm{IM}=4]$ ) received a median [IQR] daily haloperidol dose of $3.0[2.4,4.5] \mathrm{mg}$. After adjusting for age, SOFA, and ICU day, neither an association between CYP2D6 (IM $p=.67 /$ PM $p=.25$ ) or CYP3A4 (IM $p=.44$ ) metabolizer status and serum haloperidol concentrations was found. After adjusting for age, SOFA, and ICU day, neither an association between daily haloperidol dose $(p=.77)$ or ICDSC score $(p=.13)$ and serum haloperidol concentrations was found. No patient experienced QTc interval prolongation ( $\geq 500 \mathrm{~ms})$.

Conclusions: This pilot study, the first to evaluate the pharmacogenomic response of low-dose haloperidol when used to treat delirium in the ICU, suggests CYP2D6/CYP3A4 metabolizer status does not affect the serum haloperidol concentrations.
\end{abstract}

(c) 2020 Elsevier Inc. All rights reserved.

\section{Introduction}

Haloperidol is frequently administered to critically ill adults to prevent and/or treat delirium despite evidence from randomized, controlled trials that it neither prevents or resolves delirium nor improves important outcomes like mortality or post-intensive care unit (ICU) cognition [1-5]. The authors of the REDUCE trial where intravenous haloperidol (up to $8 \mathrm{mg}$ daily) was administered to prevent delirium in the ICU postulate low haloperidol serum concentrations may have influenced the lack of benefit with haloperidol they observed [6].

\footnotetext{
* Corresponding author.

E-mail addresses: z.trogrlic@erasmusmc.nl (Z. Trogrlić), m.vanderjagt@erasmusmc.n (M. van der Jagt), r.osse@erasmusmc.nl (R.J. Osse), j.devlin@northeastern.edu (J.W. Devlin), d.nieboer@erasmusmc.nl (D. Nieboer), b.koch@erasmusmc.nl (B.C.P. Koch), r.vanschaik@erasmusmc.nl (R.H.N.van Schaik),n.hunfeld@erasmusmc.nl (N.G.M. Hunfeld).
}

Haloperidol is metabolized by both the CYP2D6 and CYP3A4 isoenzyme systems [7]. Polymorphisms to either isoenzyme are common in the general population and vary by race. Among healthy adult Caucasians, extensive, intermediate and poor metabolizer prevalence is $50 \%$, $40 \%$ and $10 \%$, respectively, for CYP2D6 and 55\%, 32\% and $8 \%$ for CYP3A4 [8]. While critical illness may alter the metabolizer status of these isoenzymes, the prevalence of CYP2D6 and CYP3A4 polymorphism in the ICU remains unclear. Importantly, commonly administered ICU medications compete with haloperidol for the same CYP2D6/3A4 metabolic pathway or may act as CYP2D6/3A4 inducers or inhibitors [9]. Pharmacogenomic variability may therefore be an important contributor to haloperidol response when it is used to treat delirium in the ICU.

Although the pharmacogenomic characteristics of haloperidol have been evaluated in patients with major psychiatric disorders [10], these properties have not been evaluated in critically ill adults receiving low-dose haloperidol for the treatment of delirium $[1,4]$. This data is 
important when identifying the optimal dose of haloperidol that should be evaluated in future ICU delirium treatment trials and may help guide clinicians seeking to individualize haloperidol dosing for their patients clinically-important delirium symptom(s) (e.g., agitation) [11]. We therefore sought to characterize the pharmacogenetic characteristics of low-dose haloperidol in critically ill adults with delirium.

\section{Material and methods}

\subsection{Study design and population}

This single-center, pilot study was conducted in the adult intensive care units at Erasmus University Medical Centre, Rotterdam, NL. The study was approved by the Institutional Review Board and informed consent was obtained from each patient's legally authorized representative given all patients had delirium. A convenience sample of adults with delirium [Intensive Care Delirium Screening Checklist (ICDSC $\geq 4$ ) [12], expected to be admitted to the ICU $\geq 48 \mathrm{~h}$, and administered intravenous (IV) haloperidol according to an institutional delirium treatment protocol were evaluated for study participation between October 2014 and April 2017. Patients were excluded if they had been treated with haloperidol in the $24 \mathrm{~h}$ prior to ICU admission, had end stage liver failure or acute alcohol withdrawal, were admitted because of an acute neurologic injury, had a history of severe dementia, parkinsonism and/or psychosis, had a baseline QTc interval $\geq 450 \mathrm{msec}$, or were receiving a medication with the potential to induce either CYP2A6 and/ or CYP3A4 concentrations (i.e., bosentan, carbamazepine, efavirenz, etravirine, lopinavir, nevirapine, phenobarbital, phenytoin, rifabutin, ritonavir, rifampicin).

\subsection{Data collection}

The following baseline data was collected: gender, age, APACHE III score, body mass index (BMI), serum blood urea nitrogen, ICDSC score, QTc interval (based on ECG evaluation) and CYP2D6 and CYP3A4 isoenzyme concentrations. The following daily data was collected: daily change in ICDSC score, total dose of haloperidol administered, SOFA (Sequential Organ Failure Assessment) score, QTc interval, ICU use of medications known to inhibit CYP2D6 (i.e., amiodarone, cimetidine, fluoxetine, metoclopramide, metoprolol, paroxetine, and propranolol) and/or CYP3A4 (i.e., alprazolam, amiodarone, aripiprazole, clozapine, diazepam, erythromycin, fluconazole, melatonin, midazolam, quetiapine, risperidone, verapamil, voriconazole, zolpidem, and zopiclone) activity [13,14].

Both delirium screening (by the ICU bedside nurse using the ICDSC every $8 \mathrm{~h}$ ) and reduction efforts were well-established in all study ICUs $[15,16]$. The investigative team conducted regular spot-checks of nurse ICDSC assessments, offering additional training to nurses when required. The ICU delirium treatment protocol advocated haloperidol treatment for all patients who developed delirium at a starting dose of $1 \mathrm{mg}$ IV q $8 \mathrm{~h}$ [ $0.5 \mathrm{mg}$ IV q $8 \mathrm{~h}$ if age $\geq 80$ years old; $2 \mathrm{mg}$ IV q $8 \mathrm{~h}$ if agitation present] within $8 \mathrm{~h}$ of delirium detection. If delirium was still present 24 h later the IV haloperidol was increased by $0.5 \mathrm{mg}$ to a maximum of 2 $\mathrm{mg}$ IV q8h. The haloperidol dose was decreased when the ICDSC was $\leq 3$ for more than $24 \mathrm{~h}$, and was stopped when the ICDSC was $\leq 3$ for more than $48 \mathrm{~h}$.

CYP3A4 and CYP2D6 patient genotyping was performed using Autogenomics INFINITY genotyping platform (Carlsbad, USA) and detected CYP2D6 ${ }^{*} 2(2850 \mathrm{C}>\mathrm{T}),{ }^{*} 2 \mathrm{~A}(-1584 \mathrm{C}>\mathrm{G}),{ }^{*} 3(2549 \mathrm{G}>\mathrm{A})$, ${ }^{*} 5$ (deletion), ${ }^{*} 6$ (1707delT), ${ }^{*} 7(2935 \mathrm{~A}>\mathrm{C}),{ }^{*} 8(1758 \mathrm{G}>\mathrm{T}),{ }^{*} 9$ (2615_2617delAAG), ${ }^{*} 10(100>\mathrm{T}),{ }^{*} 12(124 \mathrm{G}>\mathrm{A}),{ }^{*} 14(1758 \mathrm{G}>\mathrm{A})$, ${ }^{*} 17(1023 \mathrm{C}>\mathrm{T}),{ }^{*} 29(1659 \mathrm{G}>\mathrm{A})$ and gene duplication and for CYP3A4 variants *1B $(-392 \mathrm{~A}>\mathrm{G}),{ }^{*} 2(15,713 \mathrm{~T}>\mathrm{C}){ }_{-},{ }^{*} 3(23171 \mathrm{C}$ $>\mathrm{T}),{ }^{*} 12(21896 \mathrm{C}>\mathrm{T}),{ }^{*} 17(15,615 \mathrm{~T}>\mathrm{C})$ and ${ }^{*} 22(15389 \mathrm{C}>\mathrm{T})$ (www.pharmvar.org). For each isoenzyme, patients were classified according to the number of active enzyme alleles present: poor metabolizers (PM; two defective alleles), intermediate metabolizers (IM, 2 decreased activity alleles or 1 active and 1 inactive allele), extensive metabolizers (EM) and ultra-rapid metabolizers (UM, gene duplication positive in absence of a CYP2D6 null allele).

Blood samples for serum haloperidol concentration evaluation were drawn from an arterial line before the administration of each morning dose on days $2,3,4,5$, and 6 (end of study) or until haloperidol was stopped due to protocol criteria, death or ICU discharge. Each blood sample was immediately sent to the hospital pharmacy laboratory, centrifuged, and the serum was stored at $-80^{\circ} \mathrm{C}$ until haloperidol quantification using validated, FDA-approved, liquid chromatography-mass spectrometry methods [17]. All serum concentrations were corrected for the most recent haloperidol dose administered.

\subsection{Data analysis}

A prospectively collected convenience sample of 20 patients was chosen given the study's pilot nature and the lack of available published ICU data to provide a standard deviation estimate for any of the outcomes evaluated. Two additional patients were enrolled due to concerns that haloperidol serum concentrations might not be able to be drawn in all patients.

The primary analysis focused on evaluating pharmacogenomic response. The decision for what to adjust for was made in advance. A linear mixed model was constructed that adjusted for age, admission SOFA score and ICU day to evaluate the association between CYP2D6 metabolizer status (or CYP3A4 metabolizer status) and daily serum haloperidol trough concentrations. To evaluate pharmacokinetic response, a third linear mixed model was constructed that adjusted for age, SOFA and ICU day to evaluate the association between daily haloperidol dose and daily serum haloperidol trough concentrations. To evaluate pharmacodynamic response, a fourth linear mixed model was constructed that adjusted for age, SOFA and ICU day to evaluate the association between the highest ICDSC score (i.e. delirium severity) and daily serum haloperidol trough concentrations. Given concerns about model overfitting, neither CYP2D6 or CYP3A4 metabolizer status were incorporated into these last two models. Haloperidol safety was evaluated by comparing the QTc interval between days and the proportion of patients where it was prolonged ( $\geq 500 \mathrm{msec}$ ). Data was presented as percentages, median (IQR) or mean (SD). Two-sided $p$ values $<.05$ were considered statistically significant. Outliers were excluded from analysis. All analyses were performed using $\mathrm{R}$ (additional packages: foreign, lme4, and rms; R Foundation for Statistical Computing, Vienna, Austria; http://www.R-project.org/).

\section{Results}

\subsection{Baseline characteristics and clinical outcomes}

Twenty-two patients (100\% Caucasian, 55\% male, 67 [IQR 60, 70] years old, BMI 27 [IQR 18, 39] kg/m², APACHE III 81 [IQR 76, 99], serum BUN $18 \pm 13 \mathrm{mmol} / \mathrm{L}$ ) were enrolled. The primary reason for ICU admission included: surgery $(7,32 \%)$, respiratory failure $(3,14 \%)$, sepsis $(3,14 \%)$ and vascular aneurysm $(2,9 \%)$. The median length of ICU stay was $16[2,63]$ days. Eleven patients died (50\%); six patients during the ICU stay, four after ICU discharge, and one after transfer to another hospital. Thirteen patients (59\%) completed the maximum six days of data collection. Detailed demographic and clinical outcomes for each patient is presented in $\boldsymbol{E}$-Table 1.

\subsection{Pharmacokinetic and pharmacogenomic outcomes}

The 81 trough serum haloperidol concentrations collected are presented by ICU day in Fig. 1. Seven very high serum haloperidol concentrations $(\geq 10 \mu \mathrm{g} / \mathrm{L})$ were observed in four patients. The median [IQR] serum haloperidol concentration for 81 samples was 1.9 [1.2 to 3.5] 
Table 1

Average ICDSC score, SOFA score and QTc interval on each study day.

\begin{tabular}{|c|c|c|c|c|c|c|c|}
\hline Parameter & Baseline & Day 1 & Day 2 & Day 3 & Day 4 & Day 5 & Day 6 \\
\hline ICDSC score ${ }^{\mathrm{a}}$ & $3.8 \pm 1.4$ & $5.0 \pm 1.1$ & $4.7 \pm 1.5$ & $4.9 \pm 1.6$ & $4.3 \pm 2.1$ & $4.5 \pm 1.7$ & $4.5 \pm 1.8$ \\
\hline SOFA score ${ }^{a}$ & $10.0 \pm 3.1$ & $9.0 \pm 3.8$ & $9.3 \pm 4.3$ & $10 \pm 5.1$ & $9.8 \pm 5.1$ & $8.4 \pm 5.1$ & $9.7 \pm 5.3)$ \\
\hline QTc interval (msec) $)^{a}$ & $413 \pm 34$ & $423 \pm 33$ & $425 \pm 27$ & $418 \pm 23$ & $413 \pm 20$ & $414 \pm 36$ & $423 \pm 36$ \\
\hline
\end{tabular}

ICDSC = Intensive Care Delirium Screening Checklist, SOFA = Sequential Organ Function Assessment.

a Presented as mean \pm SD.

$\mu \mathrm{g} / \mathrm{L}$; for the $72<10 \mathrm{mg} / \mathrm{dL}$ it was $<1.7[1.2$ to 2.95$] \mu \mathrm{g} / \mathrm{L}$. Serum concentrations for the four patients with at least one sample $\geq 10 \mathrm{mg} / \mathrm{dL}$ and presence of factors that may have accounted for a serum concentration $\geq 10 \mathrm{mg} / \mathrm{dL}$ are presented in E-Table 2 .

The CYP2D6 genotype analysis revealed: extensive metabolizers (EM) $(12,54 \%)$, intermediate metabolizers (IM) $(7,32 \%)$, and poor metabolizers (PM) $(3,14 \%)$. No ultra-rapid metabolizers were detected. The daily distribution of serum haloperidol concentrations based on CYP2D6 metabolizer status is presented in Fig. 2. An association between serum haloperidol serum concentration and CYP2D6 metabolizer status and was not found (IM $p=.67 / \mathrm{PM} p=.25$ ) (E-Table 3 ). The CYP3A4 genotype analysis revealed: EM $(18,82 \%)$ and IM $(4,18 \%)$. No ultra-rapid metabolizers or PMs were detected. The daily distribution of serum haloperidol concentrations based on CYP3A4 metabolizer status is presented in Fig. 3. An association between serum haloperidol concentration and CYP3A4 metabolizer status was not found (IM $p=$ 44) (E-Table 4).

Most $(18,82 \%)$ patients received 1 or more concomitant medications known to inhibit CYP2D6 and many $(13,59 \%)$ received 1 or more concomitant medications known to inhibit CYP3A4. A total of 5 (24\%) patients were administered quetiapine (E-Table 1).

\subsection{Haloperidol dose, delirium severity and QTc interval outcomes}

The average daily haloperidol dose, ICDSC score, SOFA score and QTC interval are presented in Table 1. The median [IQR] haloperidol dose administered across the six study days (See Fig. 4) was $3.0[2.4,4.5] \mathrm{mg}$ and was not different between individual study days $(p=.28)$. After adjustment for ICU day, age, daily SOFA score, an association between

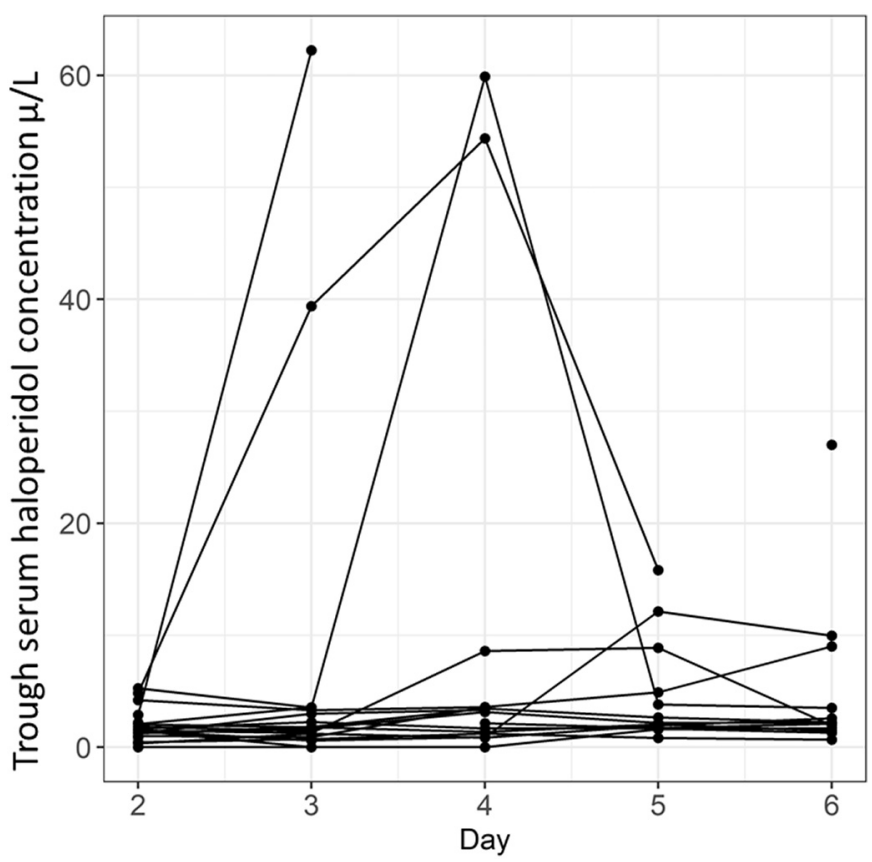

Fig. 1. Observed haloperidol trough concentrations per patient per day. haloperidol dose and serum haloperidol concentration was not found $(p=.77)$. The outputs for this linear mixed model are described in E-Table 5.

An association between the haloperidol serum concentration and delirium severity (based on ICDSC score was not found $(p=.20)$. After adjustment for ICU day, age, daily SOFA score, an association between serum haloperidol concentration and delirium severity was also not found $(p=.13)$. The outputs for this linear mixed model are described in E-Table 6.

Among the 92 ECGs performed, the QTc interval ranged from 318 and $486 \mathrm{msec}$; none exceeded $500 \mathrm{~ms}$. The average QTc interval was not different between day $1(423 \pm 33 \mathrm{~ms})$ and day $5(413 \pm 20 \mathrm{~ms})$ $(p=.48)$.

\section{Discussion}

This single-center prospective observational pilot study is the first to evaluate the pharmacogenomics of low-dose haloperidol in critically ill adults with delirium. Our results suggest that neither CYP2D6 or CYP3A4 genotype status has an important effect on serum haloperidol concentrations in critically ill adults despite a CYP3A4 extensive metabolizer status prevalence $(82 \%)$ in our critically ill cohort that is higher than that reported in healthy Caucasians (55\%) [8]. Scheduled IV haloperidol at a dose of up $2 \mathrm{mg}$ q8h does not appear to affect delirium symptoms based on the ICDSC assessments evaluated. The lack of a relationship we observed between measured serum haloperidol concentrations and the dose of haloperidol administered suggests that important factors other than age, severity of illness, ICU day of administration and CYP2D6/CYP3A4 isoenzyme status likely account

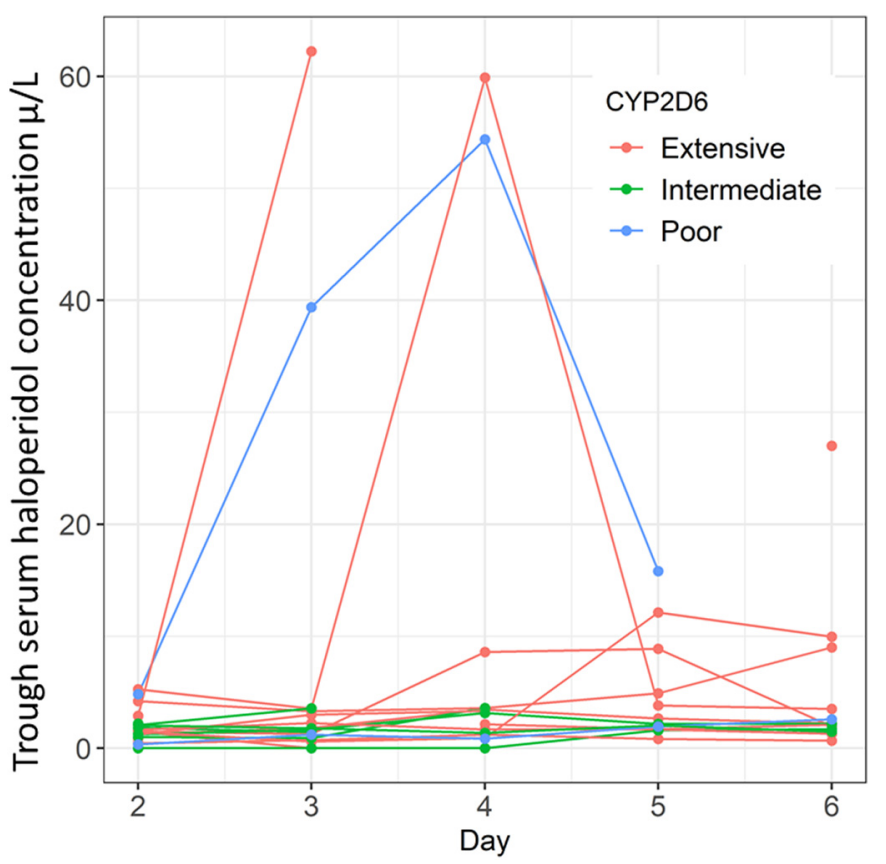

Fig. 2. Daily distribution of haloperidol serum concentrations on each study day based on CYP2D6 metabolizer status. 


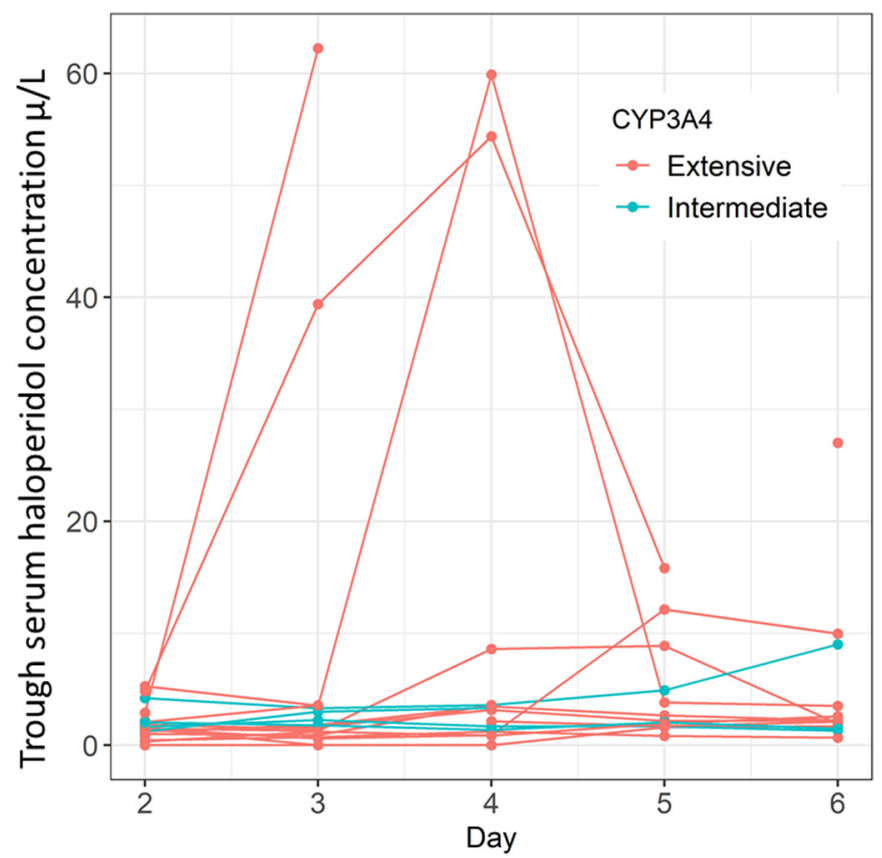

Fig. 3. Daily distribution of haloperidol serum concentrations on each study day based on CYP3A4 metabolizer status.

for the variability in serum haloperidol concentration that were observed.

While our data is consistent with a recent pharmacokinetic secondary analysis from the REDUCE trial, it is important to recognize the REDUCE analysis evaluated haloperidol use in patients without delirium and did not assess pharmacogenomic considerations like cytochrome P450 isoenzyme genotype [6]. It remains unclear if the lack of haloperidol benefit observed in our cohort is simply a result of the subtherapeutic haloperidol concentrations detected, an intrinsic lack of response of delirium to haloperidol, or related to the often fluctuating, time varying nature of delirium. Until these complex relationships are further studied, clinicians should have a low threshold level to increase the dose of haloperidol being administered to ICU patients with delirium symptoms severe enough to warrant haloperidol administration.
The results of two recent clinical trials in critically ill patients receiving low-dose haloperidol therapy have also reported a lack of effect on delirium resolution, and suggests subtherapeutic serum concentrations are an important reason for this observed lack of effect [1,4]. However, the MIND trial [3], where haloperidol was administered at a dose of 15 $\mathrm{mg} /$ day, the median [IQR] serum haloperidol trough concentration on study day two of $4.5[2.9,5.8] \mu \mathrm{g} / \mathrm{L}$. Despite patients in the MIND trial achieving a serum tough concentration nearly three-times higher than that observed in our cohort, use of haloperidol was not associated with fewer days spent with delirium. The recent MIND-USA trial found that the administration of even IV haloperidol doses (up to 20 $\mathrm{mg} /$ day) not to be associated with delirium resolution or reduced mortality. This suggests haloperidol may not have any intrinsic effect on delirium, regardless of the dose administered or the serum concentrations achieved [18].

Outside of the ICU setting, the therapeutic window for haloperidol response in patients with acute schizophrenia has been reported to range from 5.6 to $16.9 \mu \mathrm{g} / \mathrm{L}$. In this population, the recommended target concentration is 10 of $\mu \mathrm{g} / \mathrm{L}$ [10]. Whether the therapeutic dose required in schizophrenia is similar to the dose needed to treat ICU-delirium remains unclear. A recent paediatric ICU study suggests low dose haloperidol may reduce delirium despite the low serum concentrations achieved [19].

Our study has limitations. It was neither controlled nor blinded. Although patients with factors that could influence the clinical, pharmacokinetic or genomic outcomes evaluated were excluded, the heterogenous nature of any ICU population may have confounded our results. Given the variability between patients we observed, future investigations should focus on evaluating larger numbers of patients and at a greater range of haloperidol doses. Although seeking to conduct as pragmatic investigation as possible, some patients received one or more medications known to affect haloperidol serum concentrations. While haloperidol concentrations were not measured in the ICU before the first dose of haloperidol was administered, the investigators reviewed each patient record to ensure haloperidol had not been previously administered during the hospitalization. The potential reasons for the very high serum haloperidol concentrations observed in four patients were carefully evaluated. The fact that no patients had poor metabolizer status for both CYP2D6 and CYP3A4 precluded our ability to model a potential interaction between these combined poor metabolizer statuses in our analysis. Finally, in a study evaluating only 22 patients it may be possible that an association between CYP2D6/CYP3A4 isoenyzyme

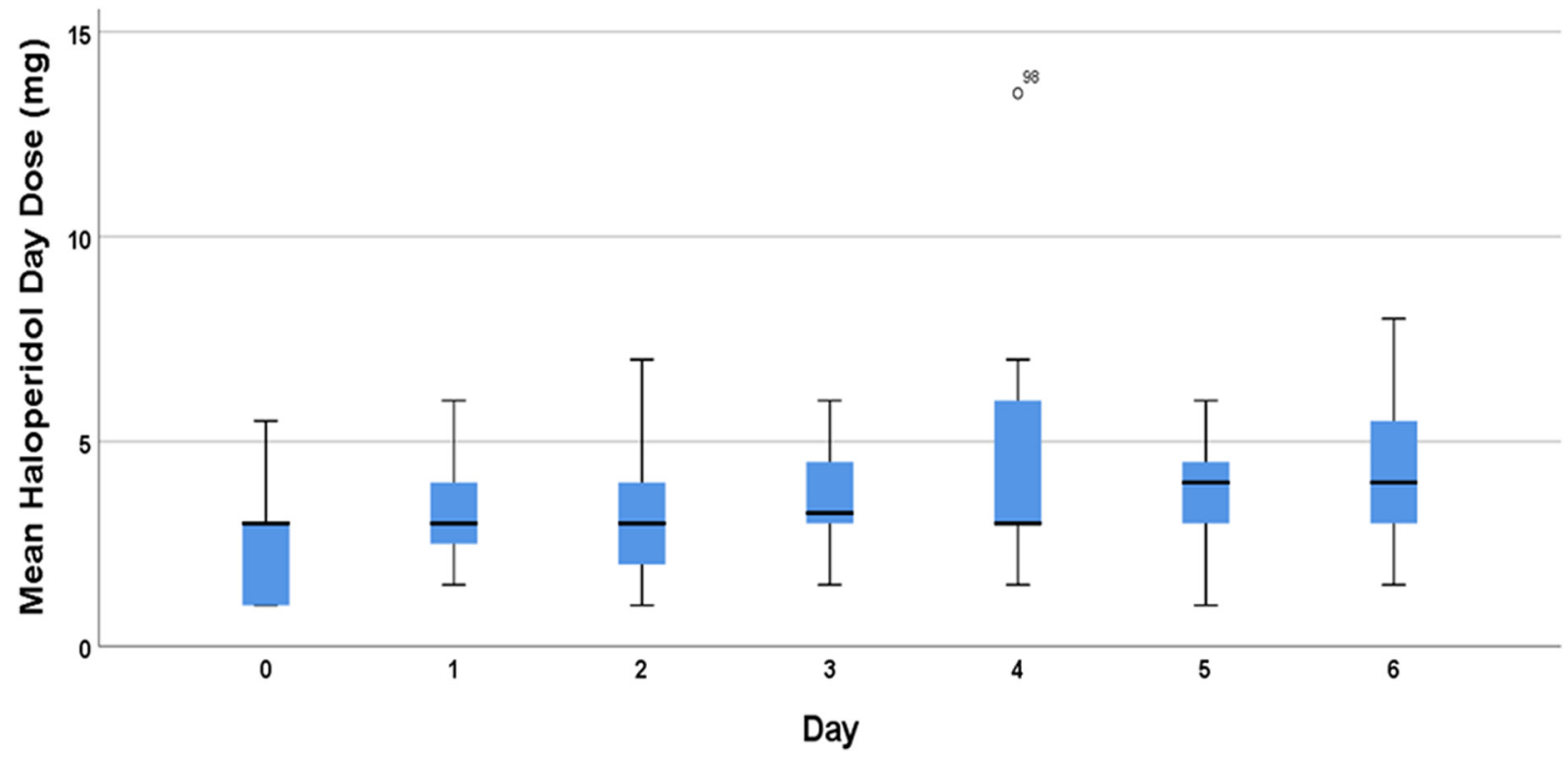

Fig. 4. Average haloperidol dose administered each study day. 
status, haloperidol dose and delirium severity and haloperidol serum concentrations do exist despite the lack of association we observed.

Future studies evaluating the pharmacokinetics and -dynamics of haloperidol in critically ill adults should seek to define clearance, areaunder-the-curve, and volume of distribution volume when testing haloperidol efficacy in critically ill adults. More extensive pharmacokinetic studies conducted at serum state concentrations and pharmacogenetic studies that control for the effects of critical illness on CYP activity are clearly indicated. Future ICU studies should focus on the presence of individual delirium symptoms with haloperidol use rather than delirium simply being either present or absent.

\section{Conclusions}

This report represents the first prospective study to evaluate the pharmacogenetic parameters of low-dose intravenous haloperidol when used for the treatment of delirium symptoms in critically ill adults. The lack of response observed with haloperidol use may be related to lower than expected serum concentrations, although we were not able to identify specific pharmacokinetic or pharmacogenomic factors accounting for this finding. It also may be possible that haloperidol is ineffective for the treatment of ICU delirium. How these factors account for the lack of clinical efficacy observed in recent randomized trials evaluating the use of low-dose haloperidol in critically ill adults remains unclear.

\section{Funding}

This research did not receive any specific grant from funding agencies in the public, commercial, or not-for-profit sectors.

\section{Declaration of Competing Interest}

The authors have no competing interests to declare.

\section{Acknowledgments}

We thank the valuable contribution of Sophie Ladage in initiating this study. We thank all ICU staff for their contribution to this study. We also thank the staff of the laboratories of Clinical Chemistry and Hospital Pharmacy, Erasmus Medical Centre for their technical contribution to this work. Finally, we thank Jan Bakker for his comments on the manuscript and Hester Lingsma for support and statistical advice.

\section{Appendix A. Supplementary data}

Supplementary data to this article can be found online at https://doi. org/10.1016/j.jcrc.2020.03.001.

\section{References}

[1] van den Boogaard M, Slooter AJC, Bruggemann RIM, Schoonhoven L, Beishuizen A Vermeijden JW, et al. Effect of haloperidol on survival among critically ill adults with a high risk of delirium: the REDUCE randomized clinical trial. JAMA 2018;319 (7):680-90.

[2] Al-Oadheeb NS, Skrobik Y, Schumaker G, Pacheco MN, Roberts RJ, Ruthazer RR, et al. Preventing ICU subsyndromal delirium conversion to delirium with low-dose IV haloperidol: a double-blind, placebo-controlled pilot study. Crit Care Med 2016;44(3): 583-91.

[3] Girard TD, Pandharipande PP, Carson SS, Schmidt GA, Wright PE, Canonico AE, et al. Feasibility, efficacy, and safety of antipsychotics for intensive care unit delirium: the MIND randomized, placebo-controlled trial. Crit Care Med 2010;38(2):428-37.

[4] Page VJ, Ely EW, Gates S, Zhao XB, Alce T, Shintani A, et al. Effect of intravenous haloperidol on the duration of delirium and coma in critically ill patients (Hope-ICU): a randomised, double-blind, placebo-controlled trial. Lancet Respir Med 2013;1(7): 515-23.

[5] Khan BA, Perkins AJ, Campbell NL, Gao S, Khan SH, Wang S, et al. Preventing postoperative delirium after major noncardiac thoracic surgery-a randomized clinical trial. J Am Geriatr Soc 2018;66(12):2289-97.

[6] van Schijndel AW, Franssen EJF, Pickkers P, Rijkenberg S, van den Boogaard M, van der Voort PHJ. Haloperidol serum concentrations in critically ill patients included in the REDUCE study. Intensive Care Med 2018;44(10):1774-5.

[7] Sychev DA, Zastrozhin MS, Miroshnichenko II, Baymeeva NV, Smirnov VV, Grishina EA, et al. Genotyping and phenotyping of CYP2D6 and CYP3A isoenzymes in patients with alcohol use disorder: correlation with haloperidol plasma concentration. Drug Metab Pers Ther 2017;32(3):129-36.

[8] Wijnen PA, Op den Buijsch RA, Drent M, Kuijpers PM, Neef C, Bast A, et al. Review article: the prevalence and clinical relevance of cytochrome $\mathrm{P} 450$ polymorphisms. Aliment Pharmacol Ther 2007;26(Suppl. 2):211-9.

[9] Spriet I, Meersseman W, de Hoon J, von Winckelmann S, Wilmer A, Willems L. Miniseries: II. Clinical aspects. Clinically relevant CYP450-mediated drug interactions in the ICU. Intensive Care Med 2009;35(4):603-12.

[10] Ulrich S, Wurthmann C, Brosz M, Meyer FP. The relationship between serum concentration and therapeutic effect of haloperidol in patients with acute schizophrenia. Clin Pharmacokinet 1998;34(3):227-63.

[11] Devlin JW, Skrobik Y, Gelinas C, Needham DM, Slooter AJC, Pandharipande PP, et al. Clinical practice guidelines for the prevention and management of pain, agitation/ sedation, delirium, immobility, and sleep disruption in adult patients in the ICU. Crit Care Med 2018;46(9):e825-73.

[12] Bergeron N, Dubois MJ, Dumont M, Dial S, Skrobik Y. Intensive care delirium screening checklist: evaluation of a new screening tool. Intensive Care Med 2001;27(5): 859-64.

[13] D.A. Flockart, Flockart, Clinical pharmacology/drug interactions/flockert table TM, Indiana University, Available from: http://medicine.iupui.edu/clinpharm/ddis/ main-table/.

[14] Kennisbank K. The Netherlands. Pharmacogenetic database. Available from: https:// www.knmp.nl/patientenzorg/medicatiebewaking/farmacogenetica/pharmacogenetics$1 ; 2018$.

[15] Ista E, Trogrlic Z, Bakker J, Osse RJ, van Achterberg T, van der Jagt M. Improvement of care for ICU patients with delirium by early screening and treatment: study protocol of iDECePTIvE study. Implement Sci 2014;9:143.

[16] Trogrlic Z, van der Jagt M, Lingsma H, Gommers D, Ponssen HH, Schoonderbeek JFJ, et al. Improved guideline adherence and reduced brain dysfunction after a multicenter multifaceted implementation of ICU delirium guidelines in 3,930 patients. Crit Care Med 2019;47(3):419-27.

[17] Administration FaD. Guidance for industry process validation: general principles and practices: Food and Drug Administration; 2011 [updated]. Available from: https:// www.fda.gov/downloads/drugs/guidances/ucm070336.pdf; January 2011.

[18] Girard TD, Exline MC, Carson SS, Hough CL, Rock P, Gong MN, et al. Haloperidol and ziprasidone for treatment of delirium in critical illness. N Engl J Med 2018;379(26): 2506-16.

[19] Slooff VD, van den Dungen DK, van Beusekom BS, Jessurun N, Ista E, Tibboel D, et al. Monitoring haloperidol plasma concentration and associated adverse events in critically ill children with delirium: first results of a clinical protocol aimed to monitor efficacy and safety. Pediatr Crit Care Med 2018;19(2):e112-9. 